\title{
Estrés Académico en las clases virtuales de los estudiantes de la Universidad Nacional José Faustino Sánchez Carrión- 2020
}

\author{
Academic Stress in the virtual classes of the students of the National University José Faustino \\ Sánchez Carrión- 2020
}

\author{
Mirtha Sussan Trejo de Ríos ${ }^{1}$,Miguel Angel Aguilar Luna Victoria, Eugenio Evaristo Andrade Flores, Carla Marina Arredondo \\ Salinas', Sthephany Lisseth Grimaldo Ramírez², Luz Daniela Guillén García
}

\begin{abstract}
RESUMEN
Objetivo: Identificar los estresores, síntomas y estrategias de afrontamiento ante el estrés académico en las clases virtuales de los estudiantes de la Universidad Nacional José Faustino Sánchez Carrión- 2020. Materiales y Métodos: Esta investigación fue de nivel descriptivo; el estudio es no experimental y transversal. La población estuvo conformada por 277 estudiantes tanto varones como mujeres de las diferentes carreras profesionales. Se utilizó la técnica documental y la encuesta mediante el instrumento del cuestionario virtual conformado por 4 ítems en las dos primeras dimensiones y 5 ítems en la última dimensión; las que fueron respondidas en forma anónima por los encuestados. Se procesó la información mediante el programa Excel, y finalmente se ha elaborado las tablas estadísticas para visualizar las características más relevantes del estudio. Resultados: El estresor más frecuente en los estudiantes que a veces se presentan es: tener tiempo limitado para hacer el trabajo que encargan los profesores/as; representado por el 50,90\%. El síntoma más frecuente en los estudiantes que a veces se presentan es: la dificultad de concentración y desgano representado por el 53,8\%. La estrategia de afrontamiento más frecuente en los estudiantes que a veces se presentan es: entretenimiento a través del internet representado por el $58 \%$. Conclusiones: Del total de estudiantes encuestados presentó un $61 \%$ nivel alto y muy alto de estrés, el $20 \%$ poco y muy poco nivel de estrés y el $19 \%$ un nivel moderado de estrés.
\end{abstract}

Palabras clave: Estrés, Estresores, Estrategias de afrontamiento, Clases virtuales.

\section{ABSTRACT}

Objective: To identify the stressors, symptoms and coping strategies in the face of academic stress in the virtual classes of the students of the National University José Faustino Sánchez Carrión- 2020. Materials and Methods: This research was descriptive level; the study is non-experimental and cross. The population consisted of 277 male and female students from different professional careers. The documentary technique and the survey were used using the virtual questionnaire instrument made up of 4 items in the first two dimensions and 5 items in the last dimension; those that were answered anonymously by the respondents. The information was processed by means of the Excel program, and finally the statistical tables have been elaborated to visualize the most relevant characteristics of the study. Results: The most frequent stressor the students that sometimes occurs is: having limited time to do the work that my teachers entrust me; represented by $50.90 \%$. The most frequent symptom in students that sometimes occurs is: difficulty in concentration and reluctance represented by $53.8 \%$. The most frequent coping strategy in students who sometimes present themselves is: entertainment through the internet, represented by $58 \%$. Conclusions: Of the total of students surveyed, $61 \%$ presented high and very high levels of stress, $20 \%$ little and very little levels of stress and $19 \%$ a moderate level of stress.

Keywords: Stress, stressors, coping strategies, virtual classes.

\section{INTRODUCCIÓN}

A inicios del 2020 se declaró una alerta epidemiológica por el brote de una nuevo enfermedad denominada COVID-19 por parte de la Organización Mundial de la Salud, debido a ello se tuvo que hacer cambios en los estilos de vida de las personas en todos los aspectos, en población juvenil tuvo un impacto en el ámbito educativo, los estudiantes universitarios emprendieron una nueva forma recibir clases fuera de las aulas, iniciando el ciclo académico las clases virtuales o remotas provocaron niveles de estrés a causa del choque en la modalidad de estudio al que estaban acostumbrados.

De acuerdo Lazarus y Folkman (1986) (Velásquez, 2020) crearon un modelo cognitivo para evaluar el estrés, definiéndolo como un conjunto de relaciones que se

Recibido 24/07/2021 Aprobado 26/08/2021

Este es un artículo de acceso abierto, distribuido bajo los términos de la Licencia Creative Commons Atribución 4.0 Internacional (http://creativecommons.org/licenses/by/4.0/)

\section{c) (i)}

Doctora en Educación, Docente de la Facultad de Ciencias de la Universidad Nacional José Faustino Sánchez Carrión, Huacho, Huaura, Lima, Perú.

Decana del colegio de Matematicos Región Huacho. ORCID: 0000-0002-2755-9950. Email: mtrejo@unjfsc.edu.pe

2 Estudiantes de la Facultad de Medicina Humana, Universidad Nacional José Faustino Sánchez Carrión, Huaura, Huacho, Lima, Perú. 
establecen entre la valoración de una situación y la capacidad para enfrentarla. Esta relación genera tensiones y manifestaciones cognitivas, emocionales y conductuales que afectan la percepción del bienestar personal frente a un posible peligro, real o imaginado. El estrés tiene una función adaptativa que busca restablecer el equilibrio a nuevas circunstancias. (Pág. 1639)

Según la revista (Quiliano Navarro Mónica, 2020) "señala que el $63 \%$ de los estudiantes presentó alguna vez este tipo de estrés, con un promedio de intensidad alta $(81,3 \%)$, lo cual puede influir de manera negativa en su estado de salud, su aprendizaje y desempeño académico" (Pág. 4)

La autora Gonzales (2020) cita a Martínez que en el año 2019 da a conocer los resultados de su investigación realizada en 6 universidades (3 nacionales y 3 privadas) de los departamentos de Lima, Arequipa y Piura, donde buscaba la relación entre el estrés académico y la adaptación a la vida universitaria. El nivel de estrés de los estudiantes fue medianamente alto con un $41 \%$, por otro lado, los factores estresantes fueron la sobrecarga de tareas y trabajos, evaluaciones de los docentes y cursos. Respecto a los síntomas generados por el estrés fueron problemas de concentración, somnolencia y cambios en el apetito.

Los alumnos universitarios de Lima presentaron una mayor intensidad de estrés a diferencia que de Piura, asimismo los estudiantes de Piura tuvieron una mejor adaptación a la vida universitaria en contraste a Lima y Arequipa. Por ende, el estrés académico y la adaptación a la vida universitaria están asociados. (Pág. 11).

En el artículo del Lovón y Cisneros (2020) mencionan que el $51,4 \%$ de los alumnos que actualmente estudian en la PUCP pensaron en retirarse parcial o totalmente del ciclo. Al preguntar el motivo, sorprende que gran cantidad de las respuestas tengan relación con afecciones en su salud mental.

Algunas de ellas alegan, incluso, que "Me lo recomendaron los psicólogos". Entre los alumnos que pensaron en retirarse y se quedaron también encontramos respuestas relacionadas a lo económico. Las respuestas en este ámbito abarcan desde la valoración negativa de las clases virtuales hasta las afecciones en los ingresos familiares causadas por la coyuntura actual. (Pág. 12).

En el periódico Español Ultima Hora (2020) nos informó que a través de una encuesta realizada entre estudiantes reveló una realidad de la que se habla desde hace semanas en la comunidad escolar. El 60,3\% de los estudiantes desde el tercer ciclo de la Escolar Básica hasta los de la Educación Media sienten estrés, miedo y ansiedad debido a la educación virtual. Esto, de acuerdo con datos dados a conocer por la Unión Nacional de Centros de Estudiantes (Unepy). "Casi 2 estudiantes de 3 están sufriendo psicológicamente por la situación producida por el Covid-19 en su educación, algo que consideran está afectando de manera importante su salud mental y la calidad de su vida en la actualidad", indicaron los jóvenes. En una consulta anterior, señalaron que el $84 \%$ del estudiantado apuntaba a que las clases virtuales durante la pandemia son malas o a medias. Desde la Unepy manifiestan que pese a que desde el Ministerio de Educación y Ciencias (MEC) les prometieron mejoras en la educación a distancia, las cosas siguen igual a menos de dos meses del fin del año escolar. (pág. 1).

De acuerdo al Periódico para la comunidad de egresados de la Universidad del Pacífico, (2020) a nivel nacional solo el $28 \%$ de ocho millones de hogares en el Perú cuenta con acceso a Internet, el 93\% de hogares del área rural y el $56,7 \%$ de hogares en el área urbana no cuentan con laptop ni computadora; siendo uno de los principales obstáculos para el aprendizaje de los estudiantes universitarios. (Pag. 12).

\section{MATERIALES Y MÉTODOS}

Esta investigación fue de nivel descriptivo, las encuestas se aplicaron de manera virtual a los estudiantes de la Universidad José Faustino Sánchez Carrión, el estudio es no experimental y transversal. La población estuvo conformada por 277 estudiantes tanto varones como mujeres de las diferentes carreras profesionales.

Se utilizó la técnica documental, que consistió en recolección de información por medio de bibliografías, revistas, medios impresos, periódicos, artículos, boletines y monografías las cuales se realizó la revisión, ordenamiento y análisis de información bibliográfica.

Se empleó la técnica de la encuesta y se elaboró el cuestionario conformado por 13 ítems con las opciones: 1 (nunca), 2 (a veces) y 3 (siempre); medida en tres dimensiones: estresores con cuatro preguntas, síntomas o reacciones con cuatro preguntas y estrategia de afrontamiento con cinco preguntas, las que fueron respondidas en forma anónima por los encuestados, las respuestas resultantes de esta aplicación fueron codificadas y analizadas con el fin de determinar claridad, coherencia y pertinencia de las mismas a fin de obtener sinceridad en sus declaraciones.

\section{RESULTADOS}

Un total de 277 estudiantes participaron en el estudio respondiendo los cuestionarios vía on line, el $68 \%$ son mujeres y el $32 \%$ son varones; sus edades estuvieron comprendidas de 17 a 29 años siendo el promedio 23 años, el estudio indicó que el $87 \%$ de los estudiantes encuestados presentan estrés y el $13 \%$ no presentan estrés; respecto al nivel de estrés: el $6 \%$ presentan muy poco, $14 \%$ poco, $19 \%$ moderado, $30 \%$ alto y $31 \%$ muy alto.

Los estresores más frecuentes en los estudiantes que a veces se presentan fueron: la sobrecarga de tareas y trabajos académicos, la forma de evaluación de los(as) 
profesores/as de las tareas, foros, proyectos, etc. y tener tiempo limitado para hacer el trabajo que encargan los profesores/as; representados por el 46,21 \%, 47,65\% y $50,90 \%$ respectivamente, tal como se aprecia en la tabla 4.

Los síntomas más frecuentes en los estudiantes que a veces se presentó fueron: alteración en el consumo de alimentos, dificultad de concentración y desgano representados por el $51,6 \%$ y $53,8 \%$ respectivamente, tal como se indica en la tabla 5.

Las estrategias de afrontamiento más frecuentes en los estudiantes que a veces se presentó fueron: logra concentrarse y establece soluciones, solicita apoyo en la familia, amigos, etc. y entretenimiento a través de los internet representados por el 54\%, 56\% y 58\% respectivamente, indicado en la tabla 6.

Tabla 1

Estudiantes encuestados por sexo

\begin{tabular}{ccc}
\hline Sexo & Frecuencia & $\%$ \\
\hline Femenino & 187 & 68 \\
\hline Masculino & 90 & 32 \\
\hline Total & 277 & 100 \\
\hline
\end{tabular}

\section{Tabla 2}

Respuestas de los estudiantes encuestados si han tenido preocupación, nerviosismo (estrés)

\begin{tabular}{ccc}
\hline Respuesta & Frecuencia & $\%$ \\
\hline $\mathrm{Si}$ & 242 & 87 \\
\hline No & 35 & 13 \\
\hline Total & 277 & 100 \\
\hline
\end{tabular}

Tabla 3

Distribución de los estudiantes encuestados por nivel de estrés

\begin{tabular}{ccc}
\hline Nivel de estrés & Frecuencia & $\%$ \\
\hline Muy poco & 16 & 6 \\
Poco & 40 & 14 \\
Moderado & 54 & 19 \\
Alto & 82 & 30 \\
\hline Muy alto & 85 & 31 \\
\hline TOTAL & $\mathbf{2 7 7}$ & $\mathbf{1 0 0}$ \\
\hline
\end{tabular}

\section{Tabla 4}

Respuestas de los estudiantes encuestados por estresores

\begin{tabular}{|c|c|c|c|c|c|c|c|c|}
\hline \multirow{3}{*}{ Síntomas } & \multicolumn{6}{|c|}{ Respuestas } & \multirow{2}{*}{\multicolumn{2}{|c|}{ TOTAL }} \\
\hline & \multicolumn{2}{|c|}{ Nunca } & \multicolumn{2}{|c|}{ A veces } & \multicolumn{2}{|c|}{ Siempre } & & \\
\hline & $\mathbf{N}^{\circ}$ & $\%$ & $\mathbf{N}^{\circ}$ & $\%$ & $\mathbf{N}^{\circ}$ & $\%$ & $\mathbf{N}^{0}$ & $\%$ \\
\hline Siento trastornos del sueño & 75 & 27 & 119 & 43 & 83 & 30 & 277 & 100 \\
\hline Sentimientos de depresión y ansiedad & 79 & 28.5 & 124 & 44.8 & 74 & 26.7 & 277 & 100 \\
\hline Dificultad de concentración y desgano & 71 & 25.6 & 149 & 53.8 & 57 & 20.6 & 277 & 100 \\
\hline Alteración en el consumo de alimentos & 54 & 19.5 & 143 & 51.6 & 80 & 28.9 & 277 & 100 \\
\hline
\end{tabular}

Tabla 5

Respuestas de los estudiantes encuestados por síntomas o reacciones

\begin{tabular}{|c|c|c|c|c|c|c|c|c|}
\hline \multirow{3}{*}{ Síntomas } & \multicolumn{6}{|c|}{ Respuestas } & \multirow{2}{*}{\multicolumn{2}{|c|}{ TOTAL }} \\
\hline & \multicolumn{2}{|c|}{ Nunca } & \multicolumn{2}{|c|}{ A veces } & \multicolumn{2}{|c|}{ Siempre } & & \\
\hline & $\mathbf{N}^{\circ}$ & $\%$ & $\mathbf{N}^{\circ}$ & $\%$ & $\mathbf{N}^{\circ}$ & $\%$ & $\mathbf{N}^{\circ}$ & $\%$ \\
\hline Siento trastornos del sueño & 75 & 27 & 119 & 43 & 83 & 30 & 277 & 100 \\
\hline Sentimientos de depresión y ansiedad & 79 & 28.5 & 124 & 44.8 & 74 & 26.7 & 277 & 100 \\
\hline Dificultad de concentración y desgano & 71 & 25.6 & 149 & 53.8 & 57 & 20.6 & 277 & 100 \\
\hline Alteración en el consumo de alimentos & 54 & 19.5 & 143 & 51.6 & 80 & 28.9 & 277 & 100 \\
\hline
\end{tabular}


Tabla 6

Respuestas de los estudiantes encuestados por estrategias de afrontamiento

\begin{tabular}{|c|c|c|c|c|c|c|c|c|}
\hline \multirow{3}{*}{ Estrategias de afrontamiento } & \multicolumn{6}{|c|}{ Respuestas } & \multirow{2}{*}{\multicolumn{2}{|c|}{ TOTAL }} \\
\hline & \multicolumn{2}{|c|}{ Nunca } & \multicolumn{2}{|c|}{ A veces } & \multicolumn{2}{|c|}{ Siempre } & & \\
\hline & $\mathbf{N}^{\circ}$ & $\%$ & $\mathbf{N}^{\circ}$ & $\%$ & $\mathbf{N}^{\circ}$ & $\%$ & $\mathbf{N}^{\circ}$ & $\%$ \\
\hline $\begin{array}{l}\text { Logra concentrarse y establece } \\
\text { soluciones. }\end{array}$ & 33 & 12 & 151 & 54 & 93 & 34 & 277 & 100 \\
\hline $\begin{array}{l}\text { Mantiene el control de sus } \\
\text { emociones }\end{array}$ & 48 & 17 & 144 & 52 & 85 & 31 & 277 & 100 \\
\hline $\begin{array}{l}\text { Realiza ejercicio físico y métodos de } \\
\text { relajación }\end{array}$ & 86 & 31 & 142 & 51 & 49 & 18 & 277 & 100 \\
\hline $\begin{array}{l}\text { Solicita apoyo en la familia, amigos, } \\
\text { etc. }\end{array}$ & 81 & 29 & 154 & 56 & 42 & 15 & 277 & 100 \\
\hline Entretenimiento a través del internet & 83 & 30 & 161 & 58 & 33 & 12 & 277 & 100 \\
\hline
\end{tabular}

\section{DISCUSIÓN}

En el trascurso de la pandemia debido a la Covid-19, los estudiantes universitarios cambiaron su estilo de aprendizaje, se obligaron a emplear nuevos métodos digitales donde se pudo evidenciar que no todos los estudiantes tenían la misma destreza frente a estos retos, sumados a las preocupaciones de cada uno ya que todos se vieron en diferentes situaciones y con muchos déficits, provocando mayor nivel de estrés que se vio a través de los distintos síntomas, por otro lado otros buscaron estrategias para apoyarse y encontrar una solución ante el estrés.

El $61 \%$ de los estudiantes encuestados presentó un nivel alto del estrés resultados diferentes a los reportados por la revista (Quiliano Navarro Mónica, 2020) con un 81,3\%. Respecto a los estresores el $46,21 \%$ presentó sobrecarga de tareas y trabajos académicos similares al estudio de la autora Gonzales Saldaña, Susan (2020) que cita a Martínez con un resultado de $41 \%$, además los síntomas de alteración en el consumo de alimentos, dificultad de concentración y desgano son semejantes a los del estudio previamente mencionado. Del mismo modo los sentimientos de depresión y ansiedad es de $44,8 \%$ diferentes al $60 \%$ de los estudiantes que menciona el periódico Español Ultima Hora (2020).

\section{BIBLIOGRAFÍA}

Alumni UP. (09 de Junio de 2020). El imperio del virus $\mathrm{n}$ a c i e n t e. O b te n ido de https://www.up.edu.pe/Documents/ALUMNI-UPjunio-2020-x-pagina.pdf

Gonzales, S. (2020). "Estrés académico en estudiantes del primer ciclo de la Escuela Académico Profesional de Enfermería de la Universidad Norbet Wener 2020". O b t e n i d o d e http://repositorio.uwiener.edu.pe/bitstream/handle/12 3456789/3790/T061_47031809_T.pdf?sequence=1\& isAllowed=y

Lovón, M. (2020). Repercusiones de las clases virtuales en los estudiantes universitarios en el contexto de la cuarentena por COVID-19: El caso de la PUCP. Propositos y Representaciones Revista de Psicologìa E d u c a t i v a , $8, \quad 12$. doi:http://dx.doi.org/10.20511/pyr2020.v8nSPE3

Quiliano, M. (2020). Inteligencia emocional y estrés académico en estudiantes de enfermería. SCielo, 4. doi:http://dx.doi.org/10.4067/s0717 95532020000100203 Última Hora . (06 de Octubre de $2020)$. El $60,3 \%$ de jóvenes sienten estrés con clases virtuales. Obtenido de https://www.ultimahora.com/el603-jovenes-sienten-estres-clases-virtualesn2907971.html

Velázquez, L. (2020). Esttrés académico en estudiantes universitarios asociado a la pandemia por Covid- 19 . Revista Digital de la Universidad Autónoma de Chiapas, IX(25), 163.

Veronica (09 de Agosto de 2020). Clases virtuales, estrés y la educación superior. Obtenido de https://medium.com/@3220191074/clases-virtualesestr\%C3\%A9s-y-la-educaci\%C3\%B3n-superiorc390a05952ec 